\title{
Health Problem and Occupational Stress among Chinese Doctors
}

\author{
Xiaojun Chen ${ }^{1,2}$, Xuerui Tan ${ }^{1}$, Liping $\mathrm{Li}^{2 *}$ \\ ${ }^{1}$ Department of Cardiovascular Diseases, The First Affiliated Hospital of Shantou University \\ Medical College, Shantou, China \\ ${ }^{2}$ Injury Prevention Research Center, Shantou University Medical College, Shantou, China \\ Email: *1pli@stu.edu.cn
}

Received December 2, 2012; revised January 9, 2013; accepted January 22, 2013

\begin{abstract}
This paper provides an overview of research into mental health problem and occupational stress among Chinese doctors in recent 10 years. It indicates that doctors in general hospitals have worse mental status. Occupational stress comes from over workload, high demanding from patients, occupational risk, effort-reward imbalance and fierce competition for job promotion. For medical staffs battling against severe acute respiratory syndrome (SARS), or working in catastrophic Wenchuan earthquake-affected areas, they have elevated stress and worrying levels of psychological distress. Post-traumatic stress disorder (PTSD) is a common mental health problem among them. The most common diseases the Chinese doctors usually suffered were cervical spondylosis, hyperlipidemia, hypertension, fatty liver and hyperglycemia. It could be important for health administrators to note that mental health appears to be an increasing problem in Chinese doctors and corresponding helping measure should be made.
\end{abstract}

Keywords: Mental Health; Stress; Doctors; Physical Health; China

\section{Introduction}

China is a country with the largest population in the world. The rapid economic growth in this developing country gives rise to many social problems. Mental health is one of them but the research on individual behavior and psychology start late and behind. A study in China had found that there were serious mental health problems in the occupational population in China [1]. Doctor is an occupation coping with the cure of patients and directly confronts severe illness and death. The huge population base in China results in an increasing number of patients. These facts seem to aggravate the occupational stress of Chinese doctors. The ratio of doctors to general population in China is 1:735, considerably lower than that in western countries (1:280 - 1:640) [2]. Large population basis, increasing health consciousness and overload working have made doctors exhausted with dealing with patients. Consequently, they find it very difficult to give enough care and time in explanation to patients, then it may easily leads to a tensional doctorpatient relationship, which aggravates the pressure and mental health among doctors [3]. However, few studies have been concentrated on the assessment of mental health and its related factors among Chinese doctors.

\footnotetext{
${ }^{*}$ Corresponding author.
}

Only several related research on nurses in English version can be found. Therefore, an updated English review of studies in Chinese doctor is expected to put it in the research of international literatures on mental health. This article summarized the published studies on mental health in Chinese doctor. It presents studies of doctors' health problems, in particular mental health problems and occupational stress studies. In conclusion information is given about the intervention suggestion.

\section{Methods}

This literature review using PubMed database and Chinese journal database searched with key words "doctor" "physician" and "medical staff". From retrieved article the relevant keywords "mental health", "mental disorder", "psychological problem" were identified and researched. Publications from the past 20 years were included with priority given to studies in recent 10 years. Literatures must be representative, concerning doctor's well-being, mental status, health-related behavior, working pressure and ways of relaxation. Studies on medical students or nurses were not included. Table 1 gives an overview of the selected studies between 2001 and 2011 published in Chinese medical journals. We lack an updated review in English of the contribution from this research programme. 


\section{Results}

There were 73 references located including investigation and review, of which 32 belong to epidemiological survey of mental health problems in medical staff. The rest of them excluded were viewpoints, ideas, proposal of helping measures. Table 1 lists the studies on mental health in doctor in Chinese medical institutions from year 2001 to 2011. There were several investigation sample exceed 1000 with the majority were between 100 - 300 interviewers. Most of them were investigating with

Table 1. Studies on mental health among chinese doctors 2001-2011.

\begin{tabular}{|c|c|c|c|}
\hline Year & Sample (N) & Measures & Major finding and conclusions \\
\hline 2011 & $\begin{array}{c}\text { Case }=257 \\
\text { Control }=300\end{array}$ & SAS, SDS, SCL-90 & Mental health of case group was worse than the control group \\
\hline 2011 & 114 & SCL-90 & Harmonious doctor-patient's relationship was good for mental health of medical staff \\
\hline 2011 & 368 & SCL-90 & $\begin{array}{l}\text { Continuing physiological education for doctors was imminent as their mental health was } \\
\text { decreasing. }\end{array}$ \\
\hline 2010 & 225 & SCL-90 & $\begin{array}{l}\text { Score of major items for doctor in rural area were higher than Chinese norm. Doctor's mental } \\
\text { health should receive enough attention }\end{array}$ \\
\hline 2010 & 239 & SCL-90 & Mental health of doctor in general dept. were obviously lower than ordinary people \\
\hline 2010 & 213 & SCL-90 & Mental health of community doctors were relatively better \\
\hline 2010 & 102 & SCL-90, SCSQ & Mental health of psychiatrist were poorer than national level \\
\hline 2010 & 109 & SCL-90 & Mental health of doctor was similar to ordinary population \\
\hline 2009 & 267 & SCL-90 & Overall mental health of doctor was good \\
\hline 2009 & 1763 & SCL-90 & $\begin{array}{l}\text { More than one third of the medical staffs are in a constant mental string state of depression, } \\
\text { compulsion and anxiety. }\end{array}$ \\
\hline 2009 & 500 & PCL-C, SAS, SDS & $\begin{array}{l}\text { Provenance of PTSD, anxiety and depression for medical personnel involved in rescue } \\
\text { working were still high one year after earthquake }\end{array}$ \\
\hline 2009 & 130 & HAMA & $\begin{array}{l}1 \text { year after disaster rescuing work, depression and anxiety of doctors were higher than } \\
\text { before. Social support was limited. }\end{array}$ \\
\hline 2009 & 262 & SCL-90 & Different level of mental disorder existed in medical staff \\
\hline 2009 & 481 & PTSD, SDS, SAS & $\begin{array}{l}\text { The prevalence of PTSD, SAS, and SDS within medical staffs who took part in rescue in the } \\
\text { disaster area after Wenchuan Earthquake was higher than in the non-disaster area. }\end{array}$ \\
\hline 2008 & 543 & MBI-GS & $\begin{array}{l}\text { The main significant predictors of exhaustion were role overload, responsibility, physical } \\
\text { environment and self care. }\end{array}$ \\
\hline 2008 & 405 & SCL-90 & Appearances of psychological problems of different nationality were different. \\
\hline 2008 & 159 & SCL-90, SAS & Scores of mental health for psychiatrist were higher than Chinese norm \\
\hline 2007 & 176 & SCL-90 & Overall mental health of medical staffs in township hospitals were good \\
\hline 2007 & 116 & SCL-90 & $\begin{array}{l}\text { Occupational risk factors have long-term negative effect on mental health of medical staff in } \\
\text { dept. of infectious disease. Intervention was necessary. }\end{array}$ \\
\hline 2006 & 126 & SCL-90 & Mental health of clinical practitioners was worse than Chinese norm. \\
\hline 2006 & 328 & SCL-90 & $\begin{array}{l}\text { there are significant mental health problems especially emergency department and the } \\
\text { surgeons in general hospital doctors }\end{array}$ \\
\hline 2006 & 282 & SCL-90, SCSQ & $\begin{array}{l}\text { Psychological health status of doctors was similar to Chinese norm. Gender, professional } \\
\text { titles were related. }\end{array}$ \\
\hline 2005 & 198 & SCL-90, SCSQ & Active and passive measure coping with depression were helping factors in mental health \\
\hline 2005 & 133 & GHQ-12, EPQ-RSC & Personality character was an influencing factor in doctor's mental health \\
\hline 2004 & 106 & SCL-90 & Physiological health surveillance should be done in medical staff after outbreak of SARS \\
\hline 2004 & 201 & SCL-90 & $\begin{array}{l}\text { Mental health scores of doctors dealing with SARS were significantly higher than Chinese } \\
\text { norm }\end{array}$ \\
\hline 2003 & 316 & SCL-90 & Mental health was good in community medical workers \\
\hline 2002 & 486 & SCL-90 & $\begin{array}{l}\text { Education of psychological promotion combined with ideological and political education } \\
\text { could strengthen mental health of young doctors }\end{array}$ \\
\hline
\end{tabular}


Symptom Checklist-90 (SCL-90) Chinese version and not all of the conclusions were inconsistence.

\subsection{General Mental Health among Doctors}

Doctor in general hospitals have worse mental status as most of their scores were higher than the Chinese norm, especially those worked in the departments of surgery, emergency and infectious disease [4,5]. The scores of somatization, interpersonal sensitivity, depression, anxiety, hostility, and paranoid ideation were significantly higher in male than female doctors [6]. Total scores of clinical doctors were significantly higher than those non-clinical medical staffs [7]. Doctors in community or township hospitals, or military hospital had better mental health. The latest cross-sectional study conducted in 7 teaching hospitals found the average standard scores of Self-Rating Anxiety Scale (SAS) for the male and female doctors were 46.8 and 46.7 [8], higher than the China norm in general population, and even higher than the level (45.36) among inhabitants in Wenchuan County after the 5.12 Sichuan earthquake [9].

The consensus suggestions were the same that attention from authorities should be paid to these medical practitioners as they were facing mounting pressure and thus the corresponding social and health services were in urgent demand. Positive relief-helping measures should be taken individually or by the institution to dealing with professional pressure.

\subsection{Occupational Stress in Medical Professional}

Occupational stress is a result of combined exposure to several factors in work environment and employment conditions [10]. The huge population in China has resulted in increasing number of patients and the health care system reform has enhanced patients' demand from doctors. The increasing numbers of patients and health consciousness in recent year have imposed heavy burdens on doctors. The burdens deteriorating their physical and mental health were multiple occupational pressures coming from over workload, high demanding from patients, occupational risk, effort-reward imbalance and fierce competition for title promotion [11]. The studies concentrated on the assessment of occupational stress and its related factors among Chinese doctors were limited. Hui Wu et al. using the Chinese version Personal Strain Questionnaire in 1587 doctors found that major factors associated with occupational stress differed between male and female doctors. Role boundary and role insufficient were the most crucial factors in male and female doctors respectively [12]. Siying Wu et al. explored the job burnout and associated variables among 543 Chinese doctors and found that the main significant predictors of exhaustion were role overload, responsibil- ity, physical environment and self care [13].

Several studies indicate a high prevalence of stress and psychological problems among doctors. It was revealed that Traditional Chinese Medicine doctors (TCM), in terms of job satisfaction, general psychological health, stress and coping were much more favorable than non TCM doctors. Additionally, the general health questionnaire (GHQ) of TCM doctors is higher than that of western medicine doctors [14]. In another research showed that doctors who experienced medium and high degree of stress accounted for $19.5 \%$ and $2.3 \%$ respectively. The highest stressors were caused by working treatment and load, professional level and medical treatment risk, which of these seriously influences their mental health [15]. In terms of working hours, a connection has been shown between sleep and mental health problems among doctors [16]. Emotional pressure and demanding patients were also related to mental health problems.

\subsection{Mental Health of Medical Staffs in Special Events}

Among the investigation researches collected, there were reports exploring the psychological status of medical practitioners involving in large-scale disaster rescuing work. One was the 2002-2003 severe acute respiratory syndrome (SARS) outbreak infected 8422 individuals leading to 916 deaths around the world [17]. China became the focus of worldwide attention when the coronavirus that causes SARS emerged. Sudden onset of SARS has the features of easy contagion, fast spread and high mortality, posing a great threat to human health. It was a major public health disaster. Post-traumatic stress disorder (PTSD) was one of the most prevalent long-term psychiatric diagnoses among medical staffs participating in war of SARS [18]. One year after the outbreak, SARS battling doctors still had elevated stress levels and worrying levels of psychological distress. The total mean score and factor scores except somatization were significantly lower in the hospital employees than the national norm [19]. The coping style for medical staff was very defective and their negative response equalization was significantly higher than the norm. Its main psychological symptoms lied in somatization, anxiety, terror and obsessive compulsion [20-21].

The other research focus was the catastrophic Wenchuan earthquake measuring 8.0 on the Richter scale occurred in Sichuan province of southwest China. During the earthquake, 69,227 people were killed, 374,643 injured, 17,923 listed as missing, and about 4.8 million were left homeless [22]. The symptoms of posttraumatic stress disorder and associated risk factors were investigated among health care workers in earthquake-affected areas in southwest China. These findings suggest that 
PTSD is a common mental health problem among health care workers in earthquake-affected areas. The prevalence of PTSD for overall was 23.3\%, anxiety was $21.6 \%$, depression was $49.9 \%$, and the anxiety plus depression was $19.54 \%$ [23]. The basic medical personnel in 6 severe disaster-stricken areas still show obvious mental distress at 14 months after the earthquake, some even have suicide attempt and symptoms of depression and PTSD [24]. The average standard score of SDS and the average crude score of SAS of 142 healthcare workers were significant higher than the national norm [25]. Abnormal of the mental health status in the medical staff was outstanding after Wenchuan earthquake. The situation should be given the enough attention.

\subsection{General Physical Health Status}

Doctor is a health care provider who practices the profession of medicine, which is concerned with promoting, maintaining or restoring human health through the study, diagnosis, and treatment of disease or injury. Doctors should have the less health problems than the rest of the population as they have the professional knowledge. However, doctor's physical health appears not as good as it thought it would be or even worse than that of the general population in China. Studies on health status of doctors in medical institution are abundant and repeated. The prevalence of chronic disease among doctors was $32.17 \%$ [26], prevalence of chronic illness increased with age growth. The analysis result was worrying and the consistence common diseases were cervical spondylosis, hyperlipidemia, hypertension, fatty liver and hyperglycemia [27-29]. Besides, female doctors also suffered from uterine fibroids. Yang Jie et al. analyzed 1723 medical staffs aged at 35 - 88 and found that the top five prevalences were hyperlipidemia (53.1\%), overweight and obesity (50.1\%), hypertension (31.2\%), fatty liver (25.4\%) and hyperglycemia (17.8\%) [30]. Another routine health check-up in 2877 medical staffs found that hyperlipidemia (45\%), fatty liver (37.5\%), hyperglycemia (11.7\%), hypertension (10.7\%) [31]. While among doctors with associate and senior professional titles, cervical spondylosis listed the top [27]. In a survey of 2423 health workers in a certain Beijing hospital found that cervical spondylosis was the most annoying disease [32]. While in hospitals in southern China, the perseverance of these chronic diseases was relatively lower. A research demonstrated that hyperlipidemia (34.6\%), Fatty liver (16.07\%), hypertension (5\%), diabetes $(4.5 \%)$ in a hospital in Hainan province [33]. Prevalence of sub-health in doctors was $66.5 \%$ and even higher with significant difference between male and female. The most common symptom was mental stress [34]. All these chronic diseases were closely related to fast-pace of work, high concentration of energy, long time overloaded and high pressure.

\section{Discussion}

In China, medical college requires 5 years for graduate course, 3 years for postgraduate course and 3 years for doctorate course. As for education, doctors started to take medical education course directly after they graduate from high school and, then, go to practice. While practicing as a doctor, some will prefer to take further education course. It is not easy to become a doctor, and it is even tougher to serve as a qualified doctor in the country with huge population. Moreover, China is undertaking health care system reform in which the focus is transforming from disease to health and from sustaining life to quality of life. The traditional disease-centered care model had been gradually replaced by the patient-centered care model.

In health care practice, patients have limited medical knowledge but impose high expectation in treatment effect. It could possibly lead to tensional doctor-patient relationship, driving doctor more frustrated. As a result, medical disputes would occur more frequently than before. Insufficient doctors increase their nightshifts and extending working hours. Working on Saturday, Sunday and even national legal holidays are often seen in doctors. Their rest time is considerably less than other occupational population. In addition to stress in hospital, doctors have their individual professional pressure. To keep up with the constantly updated medical knowledge and technology, doctor has to spent time on leaning after work. Besides, the professional promotion is fiercely competitive that young doctor need to pursuit higher educational degree and writing papers to be qualified. Senior doctors are undertaking scientific research project and teaching task for medical students. Excessive working hours and heavy workload do not exchange with high financial reward is one of the major complains for job dissatisfaction among Chinese doctors. Experiencing heavier occupational stress and lower job satisfaction may directly attribute to poor mental health in doctor.

The reform in present medical system is expected to inevitably deteriorate the mental health problems of doctors. It has been reported that, at present, most Chinese doctors suffer from depressive symptom [35]. Studies have revealed that the increased prevalence of mental health problems among doctors is most likely caused by occupational stress and individual factors. Pressure of overload working, demanding patients, life-long learning for medical knowledge and risk of medical treatment is related to their mental problems.

For doctors participating in life-threatening rescuing medial work, their mental health status is even worse. 
During the SARS epidemic, the working and living environment of the ward is isolated. Communication with the outside world is also subject to restrictions. The original pace of life and work had been disrupted, it not easy for them to adapt quickly; and also the fear of being infected by SARS virus all contribute to psychological stress factors [36]. Therefore, in the future disaster prevention, preparedness and disaster relief measures, unexpected major public health emergency response and rescue strategies and plans, psychological intervention measure should be carried out, so as to reduce the psychological damage caused by the disaster reduction and subsequent psychosocial problem [37].

In our review studies, chronic diseases of cervical spondylosis, hyperlipidemia, hypertension, fatty liver and hyperglycemia were common among Chinese doctors. It is different from that in Finnish study which chronic eczema, stomach and intestinal disorder, back complaints as the common physical disease in doctors. It could be different in another country as the work load and working condition are very different. However, either in developing countries or developed countries, doctors had to absorb the latest knowledge to improve their ability besides the routine works. Poor physical condition was able to aggravate work burden in comparison to good health.

\section{Conclusion}

Overall, this literature review shows that studies reveal a high prevalence of stress-related and mental disorders among doctors, and their physical health is not as good as people regarded. Doctors participating in the life-threatening major public health events should therefore be ensured psychiatric treatment afterward.

\section{REFERENCES}

[1] S. F. Yu, S. Q. Yao, H. Ding, L. Q. Ma, Y. Yang and Z. H. Wang, "Relationship between Depression Symptoms and Stress in Occupational Populations," Chinese Journal of Industrial Hygiene and Occupational Diseases, Vol. 24, 2006, pp. 129-133.

[2] J. Li, W. Yang and S. I. Cho, "Gender Differences in Job Strain, Effort Reward Imbalance, and Health Functioning among Chinese Physicians,” Social Science \& Medicine, Vol. 62, No. 5, 2007, pp. 1066-1077. doi:10.1016/j.socscimed.2005.07.011

[3] X. J. Feng, "Causes and Respond of Mental Health Problems in the Medical Staff," Chinese Medicine Modern Distance Education of China, Vol. 8, No. 15, 2010, pp. 152-153.

[4] H. Z. Jia, J. Shan and Y. Zhao, "A Survey on Psychological Status of Medical Staff in Infectious Department in Hengshui," Chinese Nursing Management, Vol. 11, 2007, pp. 28-29.

[5] L. Tu, X. Q. Zhang, N. Ren and H. Peng, “Current Situa- tion and Analysis of the Medical Staff'S Psychological Health in China," Medicine \& Philosophy, Vol. 30, No. 7, 2009, pp. 44-46.

[6] X. H. Xue, T. L. Zhao and J. G. Hu, "Mental Health of Doctors in General Hospital," Chinese Journal of Clinical Psychology, Vol. 14, No. 3, 2006, pp. 324-325.

[7] Y. B. Li, W. Zhong and P. Wang, "Study on Mental Health and Correlating Factors of Clinical Doctor vs NonClinical Doctor," Chinese Journal of Health Psychology, Vol. 13, No. 2, 2005, pp. 94-95.

[8] W. Sun, J. L. Fu, Y. Chang and L. Wang, "Epidemiological Study on Risk Factors for Anxiety Disorder among Chinese Doctors,” Occupational Health, Vol. 54, No. 1, 2012, pp. 1-8. doi:10.1539/joh.11-0169-OA

[9] M. Guo, Y. Gao, X. Wang and X. Jiang, "Survey of Anxiety and Depression of People during Wenchuan Earthquake," China Tropical Medicine, Vol. 19, 2009, pp. 383384.

[10] S. Y. Wu, H. Y. Li, X. R. Wang, S. J. Yang and H. Qiu, "A Comparison of the Effect of Work Stress on Burnout and Quality of Life between Female Nurses and Female Doctors," Archives of Environmental and Occupational Health, Vol. 66, No. 4, 2011, pp. 193-200. doi:10.1080/19338244.2010.539639

[11] Y. Tu, X. Y. Dou and R. Y. Yang, "On the Mental Health Survey of Doctors and Counter Measure," Journal of Yunan University, Vol. 31, No. S1, 2009, pp. 415-418.

[12] H. Wu, Y. Zhao, J. N. Wang and L. Wang, "Factors Associated with Occupational Stress among Chinese Doctors: A Cross-Sectional Survey," International Archives of Occupational and Environmental Health, Vol. 83, No. 2, 2010, pp. 155-164. doi:10.1007/s00420-009-0456-Z

[13] B. L. Lin, S. R. Gao, L. H. Cheng, Y. H. Sun and H. Luo, "Occupational Stress and Psychological Health between Traditional Chinese Medicine and Western Medicine Doctors in China," Chinese Mental Health Journal, Vol. 21, No. 11, 2007, pp. 779-782.

[14] S. Y. Wu, W. Zhu, H. Y. Li, Z. M. Wang and M. Z. Wang, "Relationship between Job Burnout and Occupational Stress among Doctors in China,” Stress and Health, Vol. 24, No. 2, 2008, pp. 143-149. doi:10.1002/smi.1169

[15] Y. J. Shi and L. F. Wang, "Doctors Professional Stress and Its Relation with Their Mental Health,” Chinese Journal of Public Health, Vol. 23, No. 5, 2007, pp. 529-531.

[16] R. Tyssen, "Health Problems and the Use of Health Services among Physicians: A Review Article with Particular Emphasis on Norwegian Studies,” Industrial Health, Vol. 45, No. 5, 2007, pp. 599-610. doi:10.2486/indhealth.45.599

[17] "Summary of Probable SARS Cases with Onset of Illness from 1 November 2002 to 7 August 2003," http://www.who.int/csr/sars/country/country2003_08_15. pdf

[18] C. Y. Lin, Y. C. Peng, Y. H. Wu, J. Chang, C. H. Chan and D. Y. Yang, "The Psychological Effect of Severe Acute Respiratory Syndrome on Emergency Department Staff,” Emergency Medicine Journal, Vol. 24, No. 1, 2007, pp. 12-17. doi:10.1136/emj.2006.035089 
[19] M. Gu, Y. Gu, Y. M. Mei, J. H. Lu and R. B. Yu, "Survey on Mental Health Status of Medical Practitioners in Comprehensive Hospitals of Jiangsu," Chinese Journal of Public Health, Vol. 24, No. 8, 2008, pp. 921-922.

[20] Z. H. Zhou, X. M. Li, K. N. Chen, X. Q. Li, Y. Dong and J. B. Zhao, "Relation of Mental Health and Coping Style for First-Line Medical Staff in SARS Battle," Chinese Journal of Behavioral Medical Science, Vol. 13, No. 3, 2004, p. 305.

[21] L. Shao, S. H. Xiao, T. Y. Cao, J. Xia and X. D. Li, "Survey on Mental Health of Young Medical Staff during SARS Period," Medical Journal of National Defending Forces in North China, Vol. 16, No. 3, 2004, p. 209.

[22] L. Wang, Y. Zhang, W. Wang, Z. Shi, J. Shen, M. Li, et al., "Symptoms of Posttraumatic Stress Disorder among Adult Survivors Three Months after the Sichuan Earthquake in China,” Journal of Traumatic Stress, Vol. 22, No. 5, 2009, pp. 444-450. doi:10.1002/jts.20439

[23] Z. Li, J. Li, Y. Liu, H. Liao, Y. Feng and X. L. Sun, “A Mental Health Survey of Medical Staffs Who Took Part in Rescue in Disaster Area after Wenchuan Earthquake,” Chinese Journal of Evidence-Based Medicine, Vol. 9, No. 11, 2009, pp. 1151-1154.

[24] W. J. Mao, T. Zhang, W. J. Min, et al., “The Association Analysis of Rural Doctor'S Mental Heath Status and Social Supports after Earthquake in Dujiangyan,” Sichuan Medical Journal, Vol. 30, No. 10, 2009, pp. 1649-1651.

[25] B. Zhou, Y. Hu, L. M. Yang, J. Xiao, L. Zheng and C. Yang, "Investigation the Mental Health Status of the Medical Staff after Wenchuan Earthquake,” Sichuan Medical Journal, Vol. 30, No. 10, 2009, pp. 1652-1654.

[26] Z. L. Guo, C. R. Zhang and C. Y. Chen, "Survey on Health Status of Medical Staff in a District in Guangzhou City,” Chinese Journal of Coal Industry Medicine, Vol. 14, No. 5, 2011, pp. 724-725.

[27] J. Tang, B. Lv, J. Sun and J. Song, "Analysis on the Health Status of Senior Medical Staff,” Chinese Journal of Health Education, Vol. 23, No. 10, 2007, pp. 784-785.

[28] Z. Y. Sun and Y. F. Wu, "Healthy Situation of the Medi- cal Staff in a Hospital from 2006 to 2008,” China Foreign Medical Treatment, Vol. 9, 2010, p. 150.

[29] L. P. Wang, Z. Y. Zhu, Y. B. Lin and D. Nie, “The Follow-Up Analysis about Circumstances of Health Physical Examination of Medical Staff," Chinese Journal of Clinical Healthcare, Vol. 9, No. 5, 2006, pp. 436-437.

[30] J. Yang, H. Zhao, H. Xin and Y. Q Liu, "Investigation of Health Check Results of Medical Staff in a Beijing Hospital in 2010," Chinese Journal of Misdiagnose, Vol. 11, No. 3, 2011, pp. 749-750.

[31] H. Xing and X. F. Wang, "Analysis of the Health Situation of the Medical Staff in a Hospital,” Hebei Medicine, Vol. 11, No. 4, 2005, pp. 337-338.

[32] L. N. Lai, L. N. Yang, W. W. Lu and Y. Dai, "Survey on Health and Working Conditions of Medical Staff Members in a Third: A Comprehensive Hospital in Beijing," Modern Hospital Management, Vol. 42, No. 3, 2011, pp. 58-60.

[33] D. L. Nie, "Results of Health Check-Up of Medical Workers,” China Tropical Medicine, Vol. 9, No. 3, 2009, p. 589.

[34] X. L. Gan, Q. Y. Chen and X. Z. Liu, "Sub-Health Status of Doctors in 3A Grade Hospital of Guangzhou and Its Characteristic Analysis," Chinese General Practice, Vol. 11, No. 9A, 2009, pp. 1573-1574.

[35] J. N. Wang, W. Sun, T. S. Chi, H. Wu and L. Wang, "Prevalence and Associated Factors of Depressive Symptoms among Chinese Doctors: A Cross-Sectional Survey," International Archives of Occupational and Environmental Health, Vol. 83, No. 8, 2010, pp. 905-911. doi:10.1007/s00420-010-0508-4

[36] Y. Cao and Y. Qi, "Psychological Status and Effect of Psychological Intervention in SARS Patients,” Chinese Journal of Nursing, Vol. 38, No. Suppl, 2003, pp. 233234.

[37] J. Gan, X. Q. Li, W. H. Zhang, C. Y. Gao, D. D. Yang, Y. N. Zhao, et al., "Relative Factors of Mental Health of AntiSARS Medical Staff,” Practical Journal of Medicine \& Pharmacy, Vol. 21, No. 1, 2004, pp. 42-43. 